\title{
Development of a Laser Driven Photocathode Injector and Femtosecond Scale Laser Electron Synchronization for Next Generation Light Sources
}

G. Le Sage, T. Cowan, T. Ditmire, J. Rosenzweig

\section{February 24, 2000}

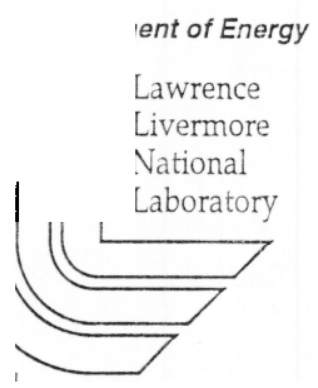




\section{DISCLAIMER}

This document was prepared as an account of work sponsored by an agency of the United States Government. Neither the United States Government nor the University of California nor any of their employees, makes any warranty, express or implied, or assumes any legal liability or responsibility for the accuracy, completeness, or usefulness of any information, apparatus, product, or process disclosed, or represents that its use would not infringe privately owned rights. Reference herein to any specific commercial product, process, or service by trade name, trademark, manufacturer, or otherwise, does not necessarily constitute or imply its endorsement, recommendation, or favoring by the United States Govermment or the University of California. The views and opinions of authors expressed herein do not necessarily state or reflect those of the United States Government or the University of Califormia, and shall not be used for advertising or product endorsement purposes.

Work performed under the auspices of the U. S. Department of Energy by the University of California Lawrence Livermore National Laboratory under Contract W-7405-Eng-48.

This report has been reproduced

directly from the best available copy.

Available to DOE and DOE contractors from the

Office of Scientific and Technical Information

P.O. Box 62, Oak Ridge, TN 37831

Prices available from (423) 576-8401

http:/ /apollo.osti.gov/bridge/

Available to the public from the

National Technical Information Service

U.S. Department of Commerce

5285 Port Royal Rd.,

Springfield, VA 22161

http://www.ntis.gov/

OR

Lawrence Livermore National Laboratory

Technical Information Department's Digital Library

http:/ /www.llnl.gov/tid/Library.html 


\title{
LDRD-ER 99-ERD-026 Final Report
}

\section{Development of a laser driven photocathode injector and femtosecond scale laser electron synchronization for next generation light sources}

\author{
PIs: Greg Le Sage (DSED), Tom Cowan (P\&ST), Todd Ditmire (LS\& T),
}

\author{
J. Rosenzweig (UCLA)
}

\section{Introduction}

A high brightness photoinjector has been developed at LLNL. This injector combined with the 100 TW FALCON laser and the LLNL $100 \mathrm{MeV}$ S-Band RF linac will enable development of a high brightness, femtosecond-scale, tunable, hard $\mathrm{x}$-ray probe for timeresolved material measurements, based on Thomson scattering. Short pulse $\mathrm{x}$-rays enable time-resolved characterization of shock dynamics, and examination of materials under extremes of pressure and temperature. Examples include Equation of State characterization on high-density materials, Crystal disorganization and re-growth in shocked and heated materials, and measurement of short time scale phase transition phenomena. Single shot evaluation, requiring high peak flux, is important for complex experiments such as probing of laser shocked actinides. A low emittance electron beam synchronized with femtosecond accuracy to an intense laser will revolutionize $x$-ray dynamics studies of materials. This project will lead development of ultrafast $x$-ray dynamics research on problems important in physics, chemistry, biology, and materials.

A schematic of the interaction between an intense laser pulse and a relativistic electron beam is shown in Fig. 1. The wavelength of the laser photons is shortened by impact with the electron bunch, which travels at nearly the speed of light. The wavelength of the scattered photons depends on the interaction angle $\Psi$. The total $x$-ray flux depends ultimately on the total number of photons and electrons that interact. In turn, the electron beam needs to be produced with high current, short length, and small transverse dimension, synchronized within less than one picosecond with the incident laser pulse. The capabilities created with the combination of the photoinjector, linac, and FALCON laser are shown also in Fig. 1.

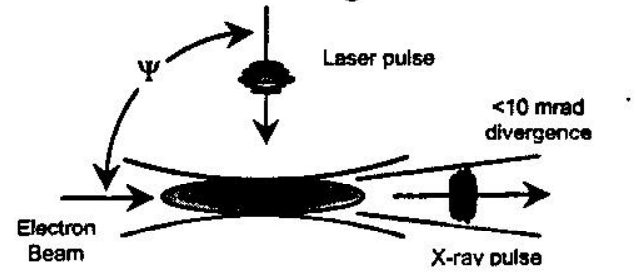
Tunable X-ray Energy \& Pulse-length: $10<\mathrm{E}_{\mathrm{x}}<300 \mathrm{keV}$ $10^{9}-10^{10} \mathrm{\gamma} / \mathrm{pulse}$ 100 fs -1 ps $4 \%-10 \%$ bandwidth

Figure 1: Laser-electron interaction 
The remainder of laser energy produced by the FALCON laser can then be applied to pump-probe experiments with the synchronized $x$-rays produced in combination with the relativistic electron beam. A schematic representation of a pump-probe measurement is shown in Fig. 2. The intense FALCON laser pulse (pump) heats or shocks a material sample, and short pulse, synchronized $x$-rays (probe) diffract from the material surface. Material properties can in this way be measured to a time resolution of order 1 ps or less.

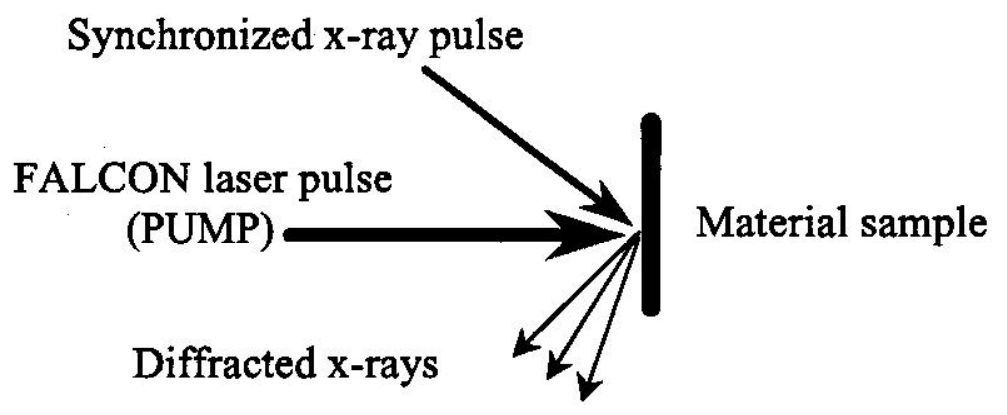

Figure 2: Pump-probe x-ray diffraction

The combination of these requirements for high peak electron current, short pulse length, and sub-picosecond synchronization necessitates a specialized type of electron injection source. The source is known as a photoinjector. The photoinjector utilizes laser-based photoemission in the presence of a high accelerating gradient. A high power microwave pulse produces the accelerating fields in the structure. A schematic of the photoinjector is shown in Fig. 3.

Microwave Power Input:

(10 MW, $3 \mu \mathrm{s})$

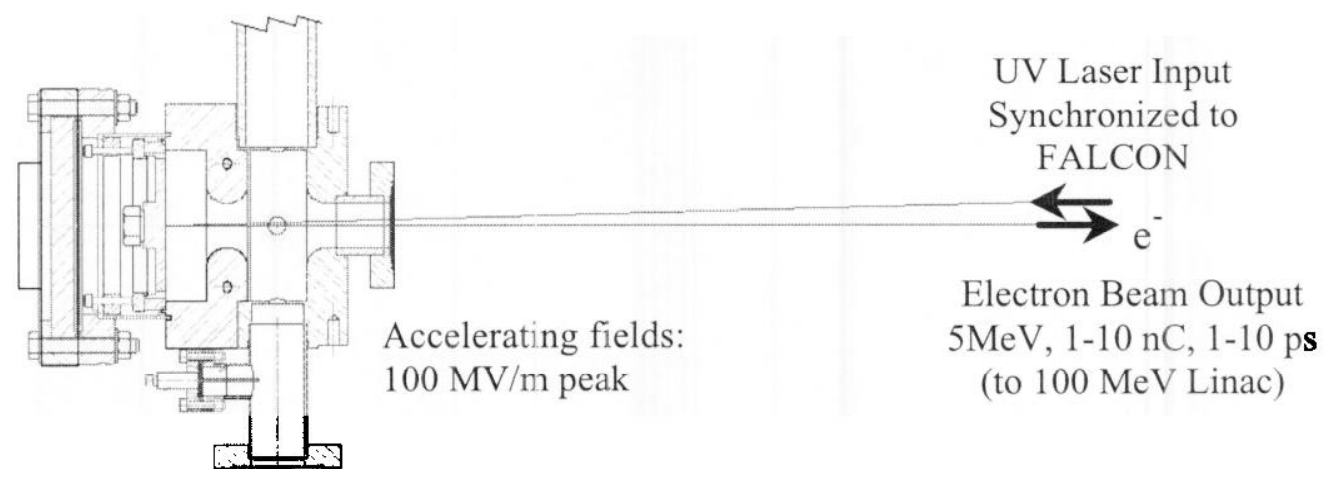

Vacuum Pumping Port 
The electron pulse is produced by a laser pulse, which in turn is synchronized to the FALCON laser. This process provides the initial laser-electron synchronization. The high gradient accelerates the beam to relativistic energies within a few centimeters, minimizing emittance growth in the beam and ultimately allowing a small final focus spot size. The photoemission process in the presence of a high accelerating gradient also allows production of high peak electron current. A schematic of the overall photoinjector-linac-FALCON laser system is shown in Fig. 4.

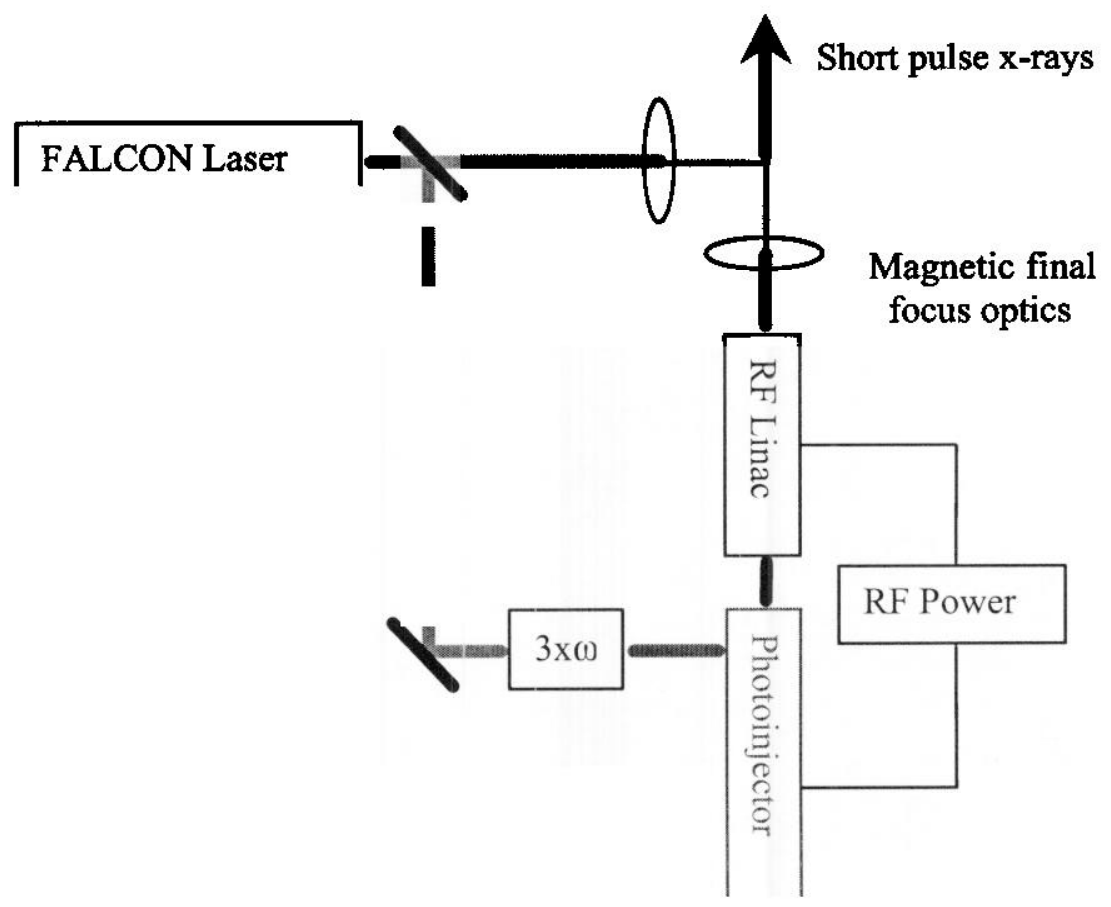

Figure 4: Thomson scattering system schematic

\section{Approach}

The goal of the photoinjector LDRD project (ERD-026) was the development of a laser driven photocathode injector (photoinjector) and femtosecond scale laser electron synchronization for the LLNL Thomson scattering project as well as other laser-electron interaction experiments leading to development of next generation light sources. The key successes of the research program were the production of a state-of-the-art photoinjector, production and measurement of a synchronized photoelectron beam, synchronization of the linac and FALCON laser systems, and analysis of new laser-electron synchronization techniques. In addition, a new electron beam diagnostic was developed which will be essential for the ultimate production of Thomson scattered $x$-rays. The path to these milestones is summarized in the following. 
The photoinjector accelerator cavity was produced in collaboration between LLNL and the UCLA department of Physics and Astronomy, with co-investigator Professor James Rosenzweig. The precision machine shop at UCLA produced the copper components that form the accelerator cavity. A photo of one of the accelerator cavity components after machining is shown in Fig. 5. All photoinjector cavity components were made from a single ingot of high isostatic-pressure (HIP) annealed oxygen-free, high conductivity (OFHC) copper. The HIP annealing process reduces grain boundary growth during further annealing and brazing steps, leading to improved performance under high RF field conditions. The OFHC certification assures maximum RF resonator quality factor (Ohmic Q). A sample of the material was examined using the glow discharge mass spectroscopy (GDMS) technique by Shiva Technologies in Morgan Hill in order to verify the purity of the copper ingot. Oxygen content was measured as $1.4 \mathrm{ppm}$ by weight.

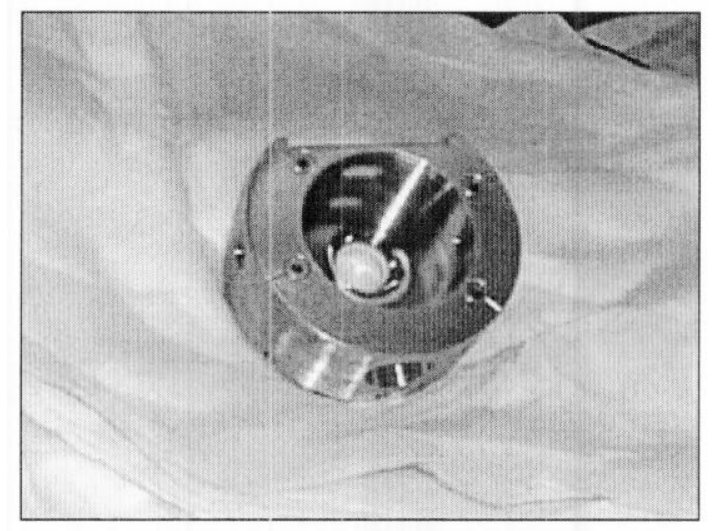

Figure 5: Precision-machined Photoinjector cavity

Once the complete set of photoinjector cavity components was manufactured, all components were "clean-fired" near the melting point of copper to remove all impurities and surface contamination. The complete set of photoinjector cavity components is shown in Fig. 6, just before the clean firing process. Clean firing is performed in a dry hydrogen environment.

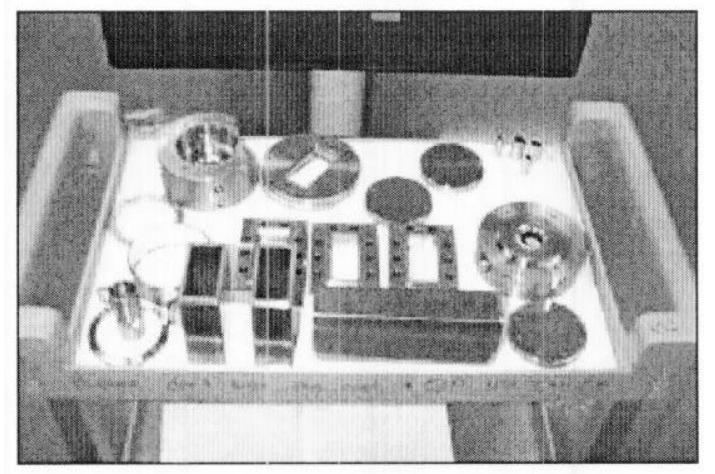

Figure 6: Photoinjector cavity parts prepared for clean-firing 
Since the maximum accelerating field gradient in the photoinjector depends strongly on the smoothness of the inner copper surfaces, the photocathode was diamond turned at LLNL. Typically, copper photocathodes are lapped with aluminum oxide paste to achieve optical smoothness. This procedure has the potential disadvantage of trapping impurities within the cathode surface, and degrading the maximum gradient capability of the structure. Diamond turning the copper with methanol used as a lubricant ensured that the surface would be very flat and very clean. The smoothness of the diamond turned region is shown in an interference plot at $632.8 \mathrm{~nm}$ in Fig. 7. The peak to valley smoothness of the diamond turned cathode region was 0.125 wave or $79.1 \mathrm{~nm}$ across the surface.

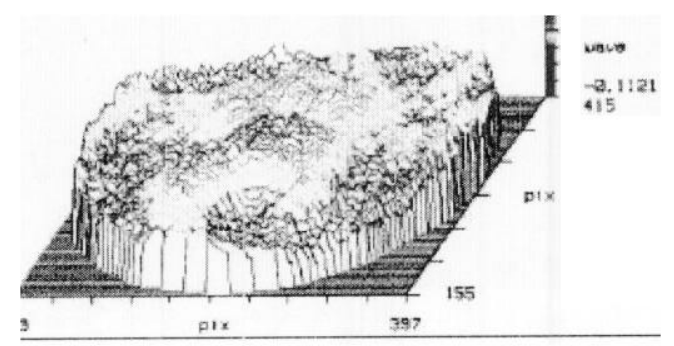

Figure 7: Diamond turned photocathode surface profile

Once the cavity components had been clean-fired, and all cavity dimensions had been inspected down to 0.0001 -inch accuracy, the parts were transported to UCLA for measurement of the RF properties of the assembled cavity. Ultimately, the cavity components were brazed together for operation under high vacuum conditions. For the initial stages of testing, the cavity components were pressed together with a specially designed clamp shown in Fig. 7A.

The purpose of the initial RF cavity tests was to properly tune the resonant frequency of the accelerator structure, and to adjust the accelerating field profile for optimum performance of the accelerated electron beam. To this end, a small dielectric bead was suspended within the accelerator cavity, as detailed in Fig. 7B (white bead shown at the cavity exit). The dielectric bead perturbs the cavity resonance in proportion to the relative field strength. In this way, a complete field amplitude map can be reconstructed. 


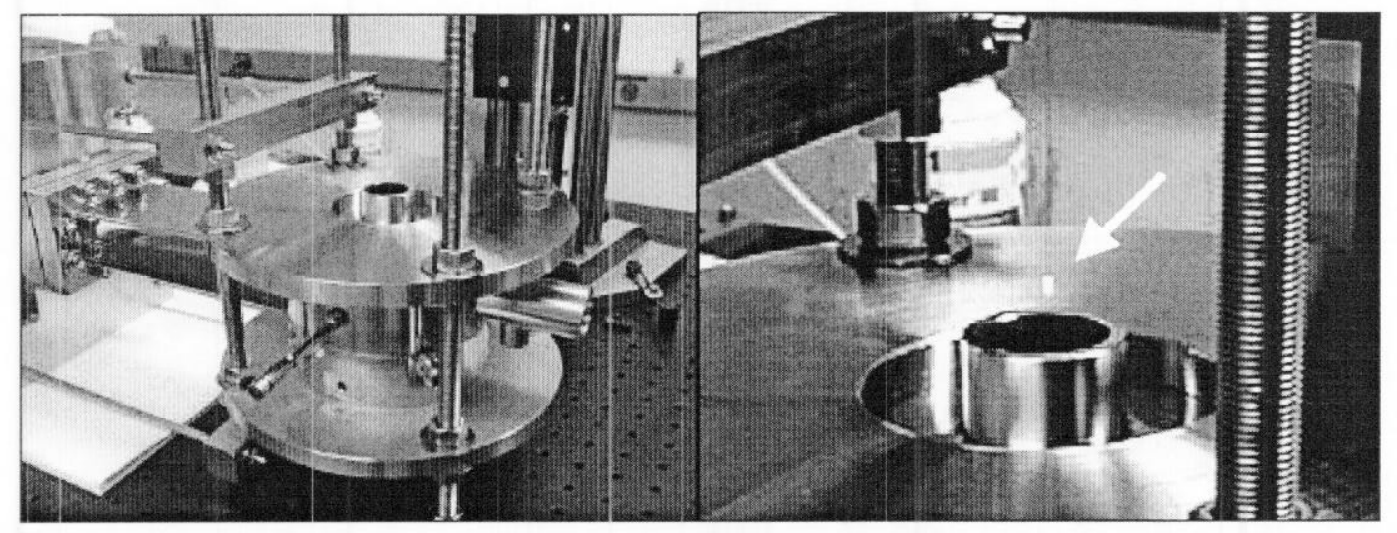

Figure 7 - A and B: Photoinjector cold test clamp

Lead experimenter Greg Le Sage is shown recording cavity RF properties in Fig. 8 using a network analyzer and this "bead-drop" apparatus. The cavity tuning process involved iterative steps of machining cuts on the inner surface of the cavity, and re-measuring the resonance and field profile.

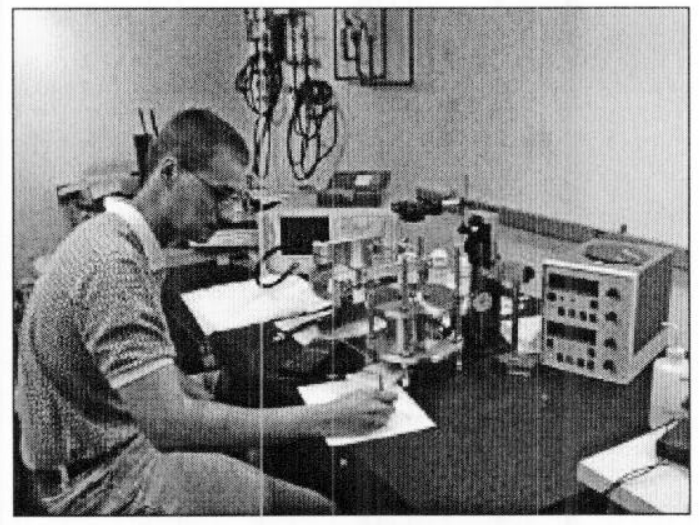

Figure 8: Photoinjector cavity RF properties are measured

The tuning cut process is in fact quite tedious and risky since damage to the precision cavity would require the part to be manufactured anew, and since removal of too much material can not be reversed. A total of seven tuning cuts were required, iterated with complete cavity measurement. Simulated field profiles guided the extent of each tuning cut and led ultimately to excellent RF properties. An accelerator cavity component fixtured in a precision lathe for a tuning cut is shown in Fig. 9. 


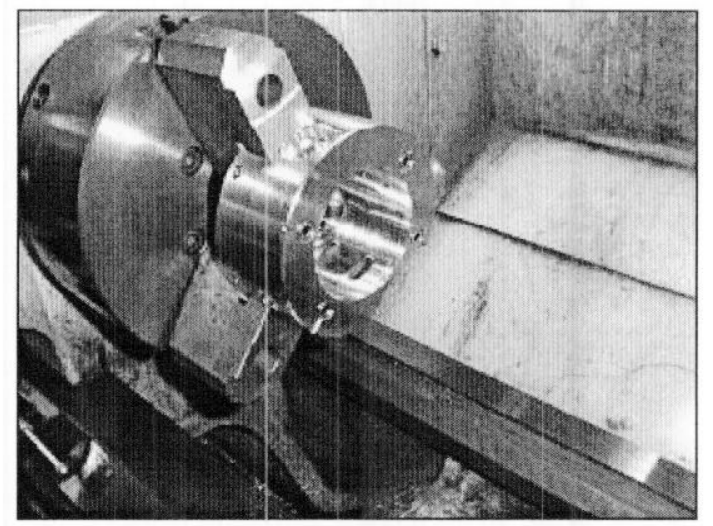

Figure 9: Photoinjector cavity tuning cuts

The final resonance profile of the accelerator cavity is shown in Fig. 10. The reflection of RF power is shown in decibel scale as a function of frequency. The key features to note are the depth of the upper-frequency resonance and the frequency spacing of the two cavity resonances. The frequency spacing of the resonances is an indicator of the field profile balance in the structure. The coupling of the RF power into the cavity is maximized at the upper frequency resonance, and indicates that the great majority of incident power is absorbed by the accelerator structure.

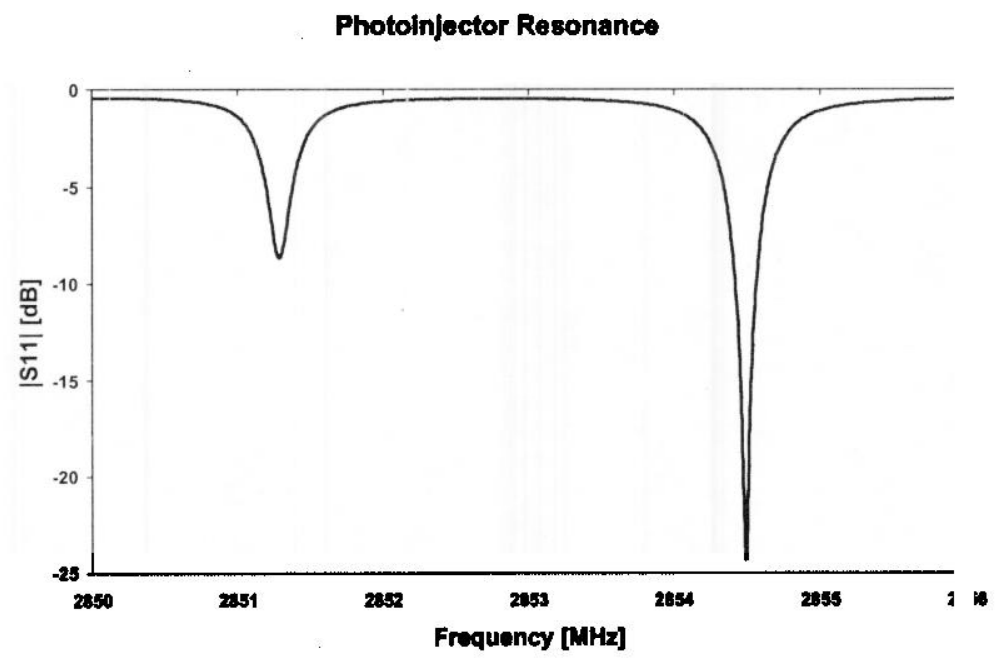

Figure 10: Photoinjector cavity resonant frequencies

The field profile measurement (bead-drop) resulted in the profile shown in Fig. 11. The data points represent the electric field amplitude inferred from resonant frequency shift induced by the presence of the dielectric bead. The solid curve is the field profile of an ideal accelerator cavity, determined through computer simulation. The field profile compared extremely well to the ideal cavity properties. 


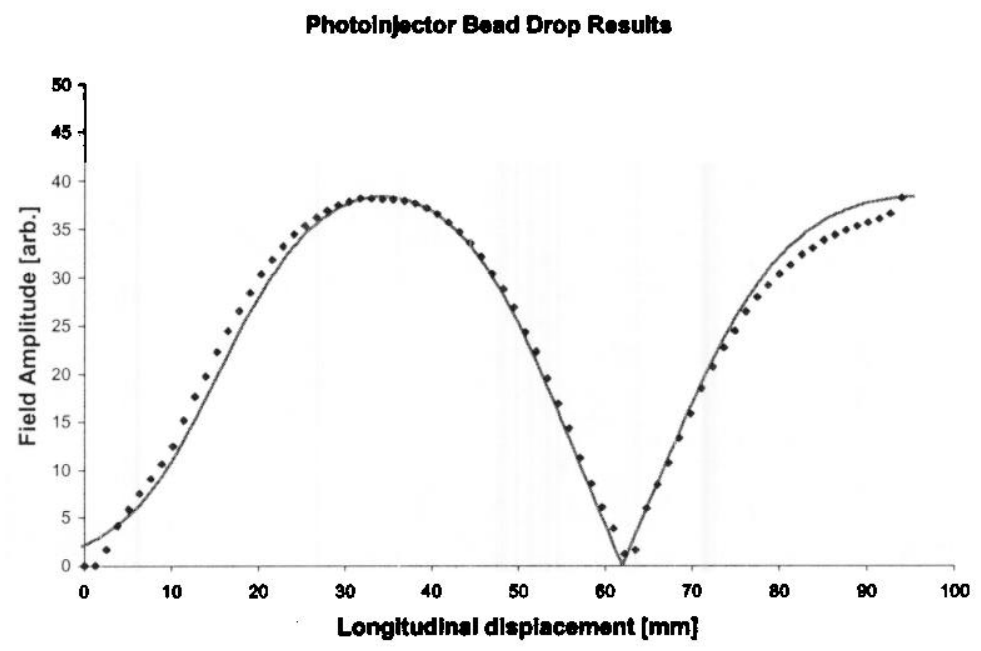

Figure 11: Photoinjector electric field profile

Once the cavity tuning procedure had been completed, the cavity parts were brazed by ALTAIR Technologies in San Carlos, under close supervision by Greg Le Sage. The final "brazing jig" is shown in Fig. 12, just after the last step in the brazing process.

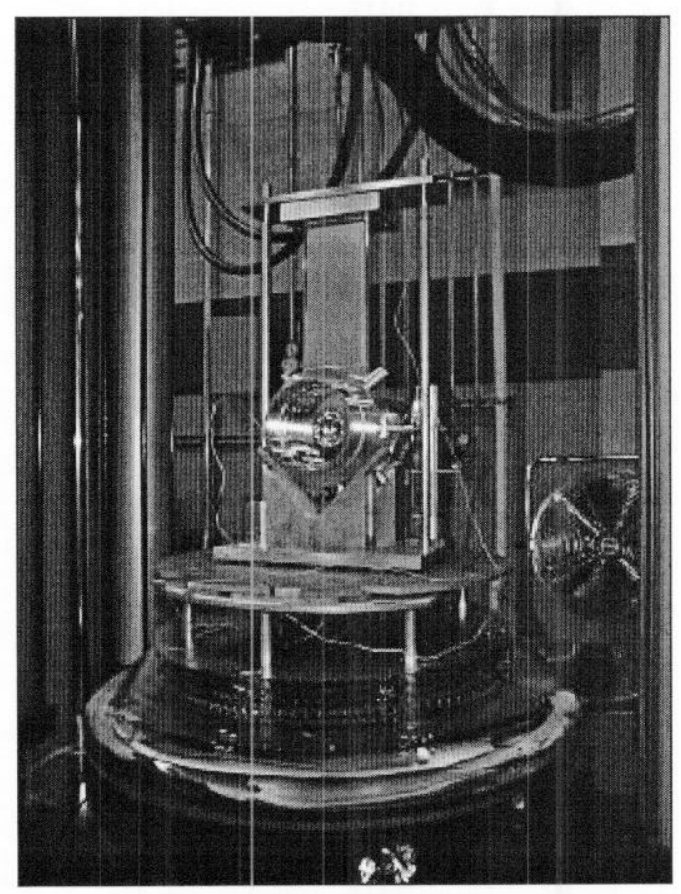

Figure 12: Photoinjector after final brazing process

Since the vacuum properties of the structure are essential to its ultimate performance, extreme care was taken in the preparation of the structure during the multi-step brazing. 
process. After final brazing and welding of vacuum flanges, the structure was baked under vacuum one final time to remove residual hydrogen which had diffused into the copper surfaces, and to remove any remaining surface contamination. The completed RF cavity and accessory components are shown in Fig. 13 after the vacuum bake-out procedure.

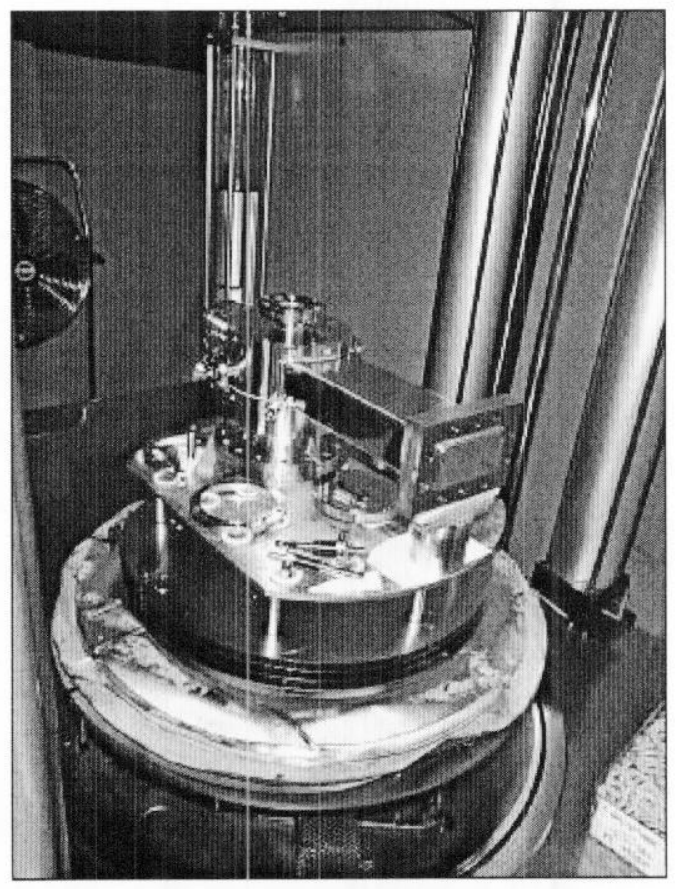

Figure 13: Final bake of completed Photoinjector

In parallel to the photoinjector construction work, a radiation shielding enclosure and electron beamline was being assembled at LLNL. A basic suite of diagnostics included beam profile and current measurement. Waveguide feed for RF power linked the photoinjector to a $21 \mathrm{MW}$ Klystron. Vacuum pumping and other ancillary systems (water and power) were arranged in anticipation of the arrival of the accelerator cavity.

A photograph of the beamline is shown in Fig. 14. The copper waveguide feed is shown entering the radiation shielding cabinet vertically. Precision fixtures for the photoinjector cavity are shown clamped to an alignment rail on top of a non-magnetic support table. Focusing solenoids (red) are shown next to the partially complete beamline.

The test beamline was constructed for the purpose of characterizing the photoinjector. Ultimately, the photoinjector will be moved to the head of the RF linac and will serve as a new injector for the electron accelerator system. The linac will boost the energy of the electron bunches from $5 \mathrm{MeV}$ (photoinjector output) up to $100 \mathrm{MeV}$. 


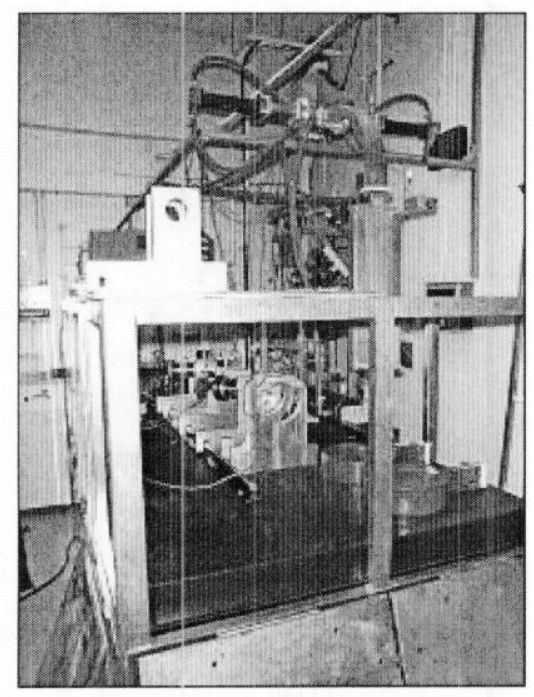

Figure 14: Photoinjector Test Beamline

The photoinjector was installed and final tuning was performed to optimize final performance. The waveguide RF power feed was attached, and the magnetic focusing components were installed. Three views of the completed test beamline are shown in Figs. $15 \mathrm{~A}, \mathrm{~B}$, and $\mathrm{C}$.
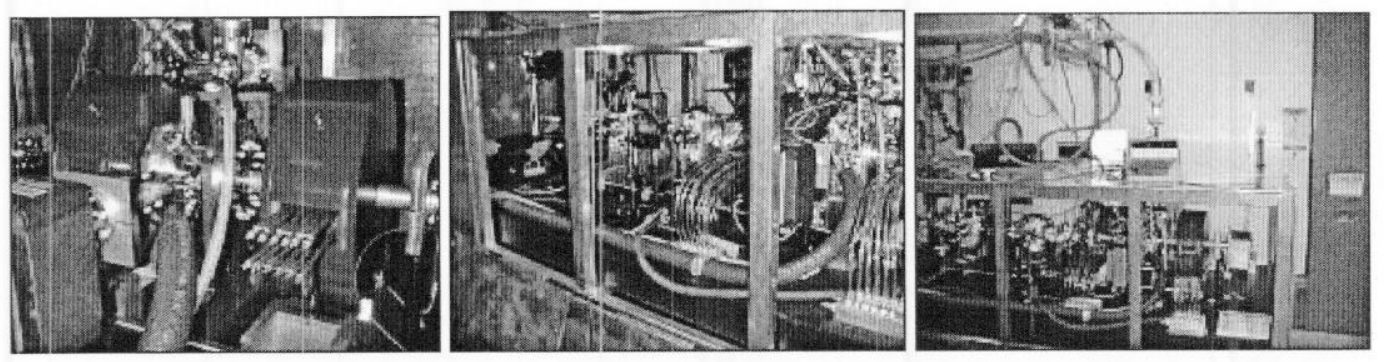

Figure 15-A, B, and C: Photoinjector beamline

High power commissioning of the photoinjector proceeded quickly, thanks to the careful handling and preparation of the cavity components. The quality factor (Ohmic $Q$ ) of the cavity was measured by comparing the time taken for the cavity to fill with RF power, and the time taken for the RF power to empty after the end of the RF drive pulse. The theoretical fit of fill and dump time is compared to measured response in Fig. 16. The $Q$ value of 13,000 verified the measurements made with a network analyzer before final installation of the photoinjector. 


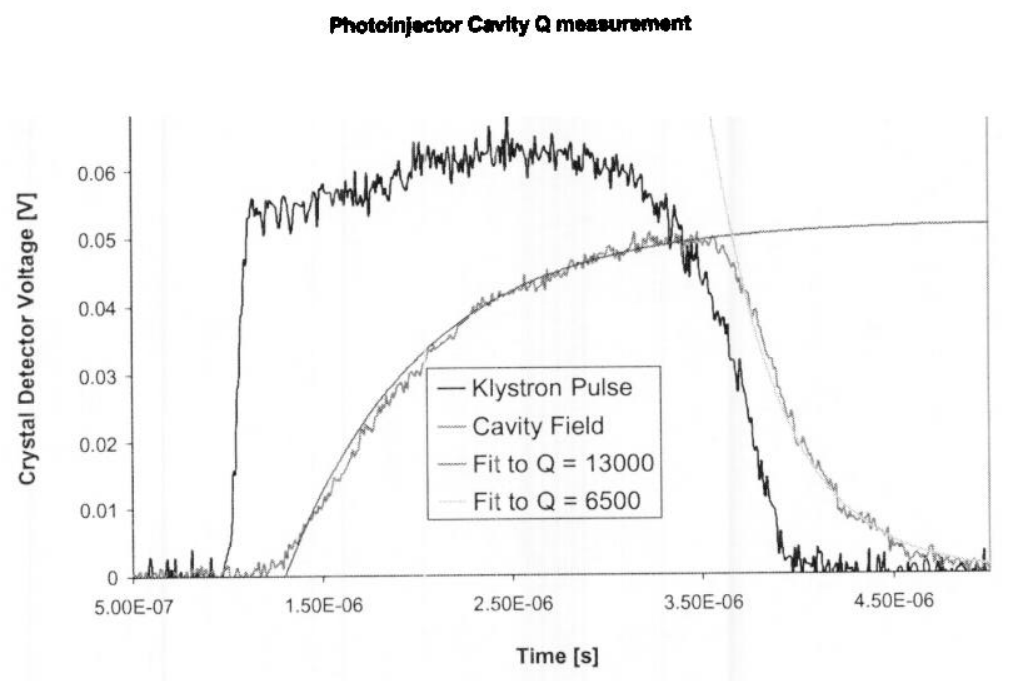

Figure 16: Characterization of photoinjector Ohmic Q

Before production of a photoelectron beam, the photoinjector cavity was characterized by "dark current" production. Dark current is the electron current produced by the accelerator cavity in the absence of UV laser pulses. As a function of RF drive power, measurement of dark current indicates the maximum field gradient that the photoinjector can support. Additionally, the peak energy of the dark current indicates the energy of the photoelectron beam that will be produced when UV is applied. The beam focussing solenoids and steering magnets were also exercised using the dark current "beam." An image of dark current production sites is produced when the solenoid is adjusted in proportion to the dark current energy, as shown in Fig. 17.

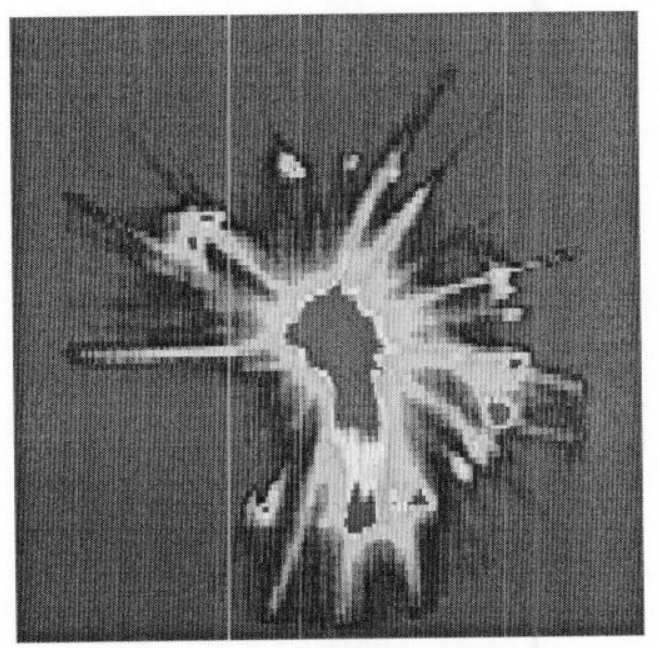

Figure 17: Photoinjector dark current 
Most recently, the UV laser system has been completed, and a photoelectron beam has been produced. In the case where the RF drive power is reduced to the point where photoelectron current dominates over dark current, the solenoid lenses can again be used to image the beam from the photocathode. An image of the photoelectron beam is shown in Fig. 18.

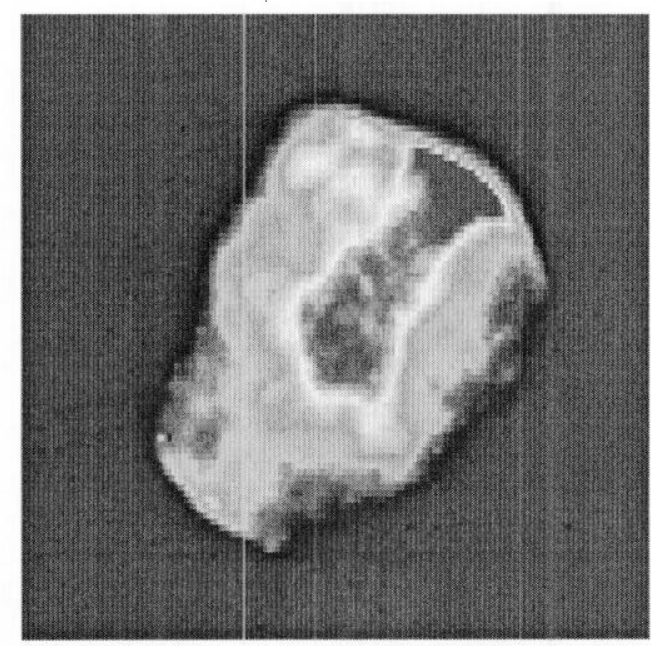

Figure 18: Photoelectron beam spatial profile

The profile of the spot indicates the transverse spatial profile of the UV laser pulse, in addition to nonuniformities in photoemission across the cathode surface. The energy of the photoelectron beam has also been characterized using the beamline steering magnets to determine beam deflection as a function of applied force. The energy of the photoelectron beam is $4.95 \mathrm{MeV}$ for a stable RF drive power level. The goal for the project was $5.0 \mathrm{MeV}$, and will be easily achievable following continued photoinjector conditioning.

The next step toward ultimate integration of the photoinjector, linac and FALCON laser is the production of Thomson scattered $\mathrm{x}$-rays using the photoinjector beam at $5 \mathrm{MeV}$. This experiment will demonstrate the synchronization the photoinjector and laser systems, as well as demonstrating that we can overcome issues of pointing and stability. A beamline and electron final-focus optics have been designed for this experiment and are currently under construction.

The $100 \mathrm{MeV}$ RF linac has been used independently for development of relativistic electron beam diagnostics based on Optical Transition Radiation. Optical Transition Radiation (OTR) is produced by a charged particle as it passes between media with different dielectric constants, for example a metallic or dielectric foil in vacuum. The angular distribution of this radiation by a single particle reveals details about its energy, position, and direction with respect to the vacuum-foil boundary. An ensemble of particles produces an angular pattern showing the superimposed contributions from each individual particle. By fitting measured angular distributions to theoretical profiles, one 
can determine statistical beam quality information. When multiple foils are arranged at specific distances in the path of a charged particle beam, a spatially coherent OTR interference pattern (OTRI) can be measured. This technique improves the sensitivity of the OTR profiles to the beam energy and divergence.

In our electron beam diagnostic experiments, optical techniques were employed to measure beam divergence as a function of transverse coordinates within the beam profile. Our experiment represented the first demonstration of this technique, and led to a publication (included in the appendix), an invention disclosure, and an R\&D 100 application.

\section{Summary}

The photoinjector integrated with the RF linac enables production of a relativistic electron beam with state-of-the-art characteristics. Combination with the FALCON laser enables unique $x$-ray probe capability. The final product of this proposal enables an operational short pulse $\mathrm{x}$-ray test-bed. Development of the photoinjector and integration with the linac also leads to a world class electron beam research program. The accomplishments supported under the photoinjector LDRD-ER project will evolve into an ongoing program of programmatically relevant materials studies.

A new proposal has also recently been submitted to the office of basic energy science (OBES) for continued funding of the Thomson scattering project at LLNL. This proposal, along with copies of the synchronization and optical beam diagnostic publications are attached in Appendix A. 


\section{Appendix A: Supporting Documents}

1. 'Synchronization of Sub-picosecond Electron and Laser Pulses" J.P. Rosenzweig, G.P. Le Sage, AIP Conf. Proc., no. 472, 795-810, (1998).

2. "Transverse phase space mapping of relativistic electron beams using Optical Transition Radiation," G. P. Le Sage, T. E. Cowan, R. B. Fiorito, and D. W. Rule, Phys. Rev. ST Accel. Beams 2, December, 1999.

3. "Time-resolved x-ray structural studies of laser-excited solids with an ultrafast, high brightness Thomson scatter $\mathrm{x}$-ray source," A proposal submitted to the OBES call: Lab 99-24 "Novel X-ray Light Sources," Todd Ditmire, Philip Bucksbaum, James Rosenzweig, Greg Le Sage, Tom Cowan, Richard Lee, Claudio Pellegrini, Bruce Remington, Justin Wark, Howard Powell. 